\title{
Sensory Evaluation Model with Unbalanced Linguistic Information
}

\author{
L. Martínez ${ }^{1}$ M. Espinilla ${ }^{2}$ L.G. Pérez ${ }^{3}$ \\ Department of Computer Science, University of Jaén, Jaén 23071, Spain
}

\begin{abstract}
The evaluation processes are used for quality inspection, marketing and other fields in industrial companies. This contribution focuses in sensory evaluation where the evaluated items are assessed according to the knowledge acquired via human senses by a panel of experts. In these evaluation processes the information provided by the experts implies uncertainty, vagueness and imprecision. The use of the Fuzzy Linguistic Approach [1] has provided successful results modeling such a type of information. Usually evaluation processes based on linguistic approaches use symmetrical and uniformly distributed linguistic term sets in order to express their preferences about the evaluated objects. However, there exist problems whose assessments need that one side of the scale overweight the other one, it means an unbalanced linguistic scale. In this contribution we present a sensory evaluation model that manages frameworks with unbalanced linguistic information.
\end{abstract}

Keywords: Sensory evaluation, unbalanced linguistic term sets, linguistic hierarchies.

\section{Introduction}

The evaluation is a complex cognitive process that involves different mechanisms in which it is necessary to define the elements to evaluate, fix the evaluation framework, gather the information and obtain an evaluation assessment by means of an evaluation process. The aim of any evaluation process is to obtain information about the worth of an item (product, service, material, etc.), a complete description of different aspects, indicators, criteria in order to improve it or to compare with other items to know which are the best ones. The information gathered in evaluation processes is usually provided by a group of individuals, called panel of experts, where each expert expresses his/her opinions about the evaluated items.
The Sensory Evaluation $[2,3,4,5]$ is an evaluation discipline where the information provided by the panel of experts, is perceived by the human senses of sight, smell, taste, touch and hearing. The experts express their opinions about the evaluated object according to their knowledge and their own perceptions. This type of information is subjective and difficult to assess quantitatively in a precise way. It is more adequate to express the information perceived by the human senses in a qualitative way by means of linguistic terms. The Fuzzy Linguistic Approach [1] provides a systematic way to represent linguistic information by means of linguistic variables in an evaluation process. The use of linguistic term sets symmetrical and uniformly distributed have provided good results in different evaluation process $[6,7,8]$, but often in evaluation processes it is common that experts need a greater level of distinction in one side of the evaluation scale (usually the positive side) than in the other one (negative side). Usually the level of goodness of an object is more relevant than how bad it is, because when it is bad the experts don't care about it. In such cases the use of Unbalanced Linguistic Information can play a key role in the evaluation process. Therefore, in sensory evaluation the use of unbalanced linguistic term sets Fig. 1, i.e., linguistic term sets with different level of discrimination on both sides to express the experts' preferences could be more appropriate in many cases.

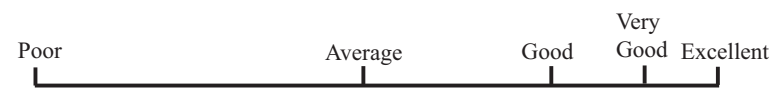

Fig. 1: Unbalanced linguistic term set of 5 labels.

The decision analysis allows people to make decisions more consistently. However, decision analysis is not an idealized theory for totally rational beings [9]. In fact, experimental evidences shows that people generally do not process information and make decisions in ways that are consistent with the decision analysis approach. Then, although decision analysis is not always followed by the deci- 
sion makers it is a suitable approach for evaluation processes.

The aim of this contribution is to present a sensory evaluation model that provides and manages a unbalanced linguistic evaluation framework such that the experts can express their opinions in it. To do so, we use a methodology to deal with Unbalanced Linguistic Information [10] based on the Linguistic Hierarchical and the 2-tuple representation model $[11,12]$ together an evaluation model based on the decision analysis [9].

This contribution is structured as follows, section 2 reviews some linguistic concepts, section 3 reviews in short a methodology to deal with unbalanced linguistic information model. Section 4 presents our proposal for the sensory evaluation model. Finally, Section 5 points out some conclusions.

\section{Linguistic background}

In this section, we review some linguistic concepts necessary to understand our proposal.

\subsection{2-tuple linguistic represen- tation model}

In [12] was presented a linguistic representation model based on linguistic 2-tuples that carries out processes of computing with words $(\mathrm{CW})$ in a precise way when the linguistic term sets are symmetrical and uniformly distributed. This model is based on the concept of symbolic translation.

The 2-tuple fuzzy linguistic representation model represents the linguistic information by means of a 2-tuple, $(s, \alpha)$, where $s$ is a linguistic label and $\alpha$ is a numerical value that represents the value of the symbolic translation.

Definition 1.[12] Let $\beta$ be the result of an aggregation of the indices of a set of labels assessed in a linguistic term set $S$, i.e., the result of a symbolic aggregation operation. $\beta \in[0, g]$, being $g+1$ the cardinality of $S$. Let $i=\operatorname{round}(\beta)$ and $\alpha=\beta-i$ be two values, such that, $i \in[0, g]$ and $\alpha \in[-.5, .5)$ then $\alpha$ is called a Symbolic Translation.

This linguistic representation model defines a set of functions to make transformations between linguistic 2-tuples and numerical values:

Definition 2.[12] Let $S=\left\{s_{0}, \ldots, s_{g}\right\}$ be a linguistic term set and $\beta \in[0, g]$ a value supporting the result of a symbolic aggregation operation, then the 2-tuple that expresses the equivalent information to $\beta$ is obtained with the following function:

$$
\begin{gathered}
\Delta:[0, g] \longrightarrow S \times[-0.5,0.5) \\
\Delta(\beta)=\left\{\begin{array}{cc}
s_{i} & i=\operatorname{round}(\beta) \\
\alpha=\beta-i & \alpha \in[-.5, .5)
\end{array}\right.
\end{gathered}
$$

where round is the usual rounding operation, $s_{i}$ has the closest index label to " $\beta$ " and " $\alpha$ " is the value of the symbolic translation.

Proposition 1.[12] Let $S=\left\{s_{0}, \ldots, s_{g}\right\}$ be a linguistic term set and $\left(s_{i}, \alpha\right)$ be a 2-tuple. There is always a function $\Delta^{-1}$, such that, from a 2-tuple it returns its equivalent numerical value $\beta \in[0, g] \subset$ $\mathcal{R}$.

$$
\begin{gathered}
\Delta^{-1}: S \times[-.5, .5) \longrightarrow[0, g] \\
\Delta^{-1}\left(s_{i}, \alpha\right)=i+\alpha=\beta .
\end{gathered}
$$

Remark 1: From definitions 1 and 2 and proposition 1 , it is obvious that the conversion of a linguistic term into a linguistic 2-tuple consist of adding a value 0 as symbolic translation:

$$
s_{i} \in S \Longrightarrow\left(s_{i}, 0\right)
$$

The 2-tuple representation model has developed a computational model presented in [12]

\subsection{Hierarchical linguistic con- text}

The hierarchical linguistic structure was used in [11] to improve the precision of the processes of CW in linguistic multi-granular contexts. It will be used in this contribution to manage the unbalanced linguistic term sets.

A linguistic hierarchy is a set of levels, where each level is a linguistic term set with different granularity from the remaining of levels of the hierarchy. Each level belonging to a linguistic hierarchy is denoted as $\mathbf{l}(\mathbf{t}, \mathbf{n}(\mathbf{t}))$, being:

1. $t$, indicates the level of the hierarchy,

2. $n(t)$, the granularity of the linguistic term set of the level $t$.

We assume levels containing linguistic terms whose membership functions are triangular-shaped, symmetrical and uniformly distributed in $[0,1]$. In addition, the linguistic term sets have an odd number of elements.

The levels belonging to a linguistic hierarchy are ordered according to their granularity, i.e., for 
any two consecutive levels $t$ and $t+1, n(t+1)>$ $n(t)$. This provides a linguistic refinement of the previous level.

From the above concepts, we define a linguistic hierarchy, $L H$, as the union of all levels $t$ :

$$
L H=\bigcup_{t} l(t, n(t))
$$

Given a $L H, S^{n(t)}$ denotes the linguistic term set of $L H$ corresponding to the level $t$ of $L H$ with a granularity of uncertainty of $n(t)$ :

$$
S^{n(t)}=\left\{s_{0}^{n(t)}, \ldots, s_{n(t)-1}^{n(t)}\right\}
$$

Generally, we can say that the linguistic term set of level $t+1, S^{n(t+1)}$, is obtained from its predecessor, $S^{n(t)}$, as:

$$
l(t, n(t)) \rightarrow l(t+1,2 \cdot n(t)-1)
$$

A graphical example of a linguistic hierarchy is shown in Fig. 2:

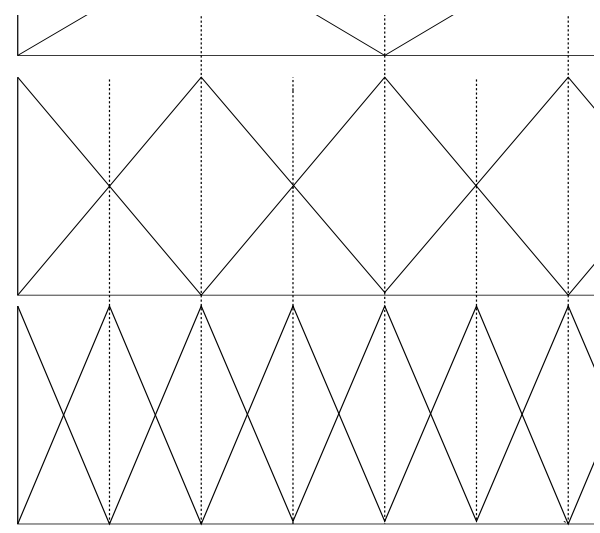

Fig. 2: Linguistic hierarchy of 3,5 and 9 labels.

In [11] was defined a transformation function between labels from different levels to carry out processes of CW in multi-granular linguistic information contexts without loss of information, it has been defined as follows:

$$
\begin{gathered}
T F_{t^{\prime}}^{t}: l(t, n(t)) \longrightarrow l\left(t^{\prime}, n\left(t^{\prime}\right)\right) \\
T F_{t^{\prime}}^{t}\left(s_{i}^{n(t)}, \alpha^{n(t)}\right)=\Delta\left(\frac{\Delta^{-1}\left(s_{i}^{n(t)}, \alpha^{n(t)}\right) \cdot\left(n\left(t^{\prime}\right)-1\right)}{n(t)-1}\right)
\end{gathered}
$$

Proposition 2.[11] The transformation function between linguistic terms in different levels of the linguistic hierarchy is bijective:

$$
T F_{t}^{t^{\prime}}\left(T F_{t^{\prime}}^{t}\left(s_{i}^{n(t)}, \alpha^{n(t)}\right)\right)=\left(s_{i}^{n(t)}, \alpha^{n(t)}\right)
$$

This result guarantees the transformations between levels of a linguistic hierarchy are carried out without loss of information.

\section{Unbalanced linguistic infor- mation}

Most of problems modeling information with linguistic assessments use linguistic variables assessed in linguistic term sets whose terms are uniform and symmetrically distributed [13]. However, there exist problems in which it is more suitable to assess their assessments by means of linguistic term sets that are not uniform and symmetrically distributed. We call this type of term sets as, unbalanced linguistic term sets. In some cases, the unbalanced linguistic information appears either due to the nature of the linguistic variables that participate in the problem, or in problems dealing with scales in which it is necessary to assess preferences with a greater granularity on a side of the scale than on the other one, as for example it happens in the sensory scale, Fig. 1.

In [10] was developed a methodology to obtain a semantic representation algorithm for unbalanced linguistic term sets that provides semantics to the linguistic terms belong to a unbalanced linguistic term set. This methodology acts in two different aims.

First, it defines an algorithm to build the semantics for an unbalanced linguistic term sets using Linguistic Hierarchies, a further and detailed description can be found in [10]. The algorithm returns a Hierarchical semantic representation, $L H(S)$ for an unbalanced linguistic term set $\mathcal{S}=\left\{s_{i}, i=0, \ldots, g\right\}$ and obtains its representation in the Linguistic Hierarchy, $L H$.

The semantic obtained $L H(S)=\left\{s_{I(i)}^{G(i)}, i=\right.$ $0, \ldots, g\}$, it is such that $\forall s_{i} \in S \exists l(t, n(t)) \in L H$ that contains a label $s_{k}^{n(t)} \in S^{n(t)}$, in such a way that $I(i)=k$ and $G(i)=n(t)$, being $I$ and $G$ functions that assign to each label $s_{i} \in S$ the index of the label that represents it in the linguistic hierarchy and the granularity of label set of linguistic hierarchy in which it is represented, respectively.

Second, the methodology defines a computational model for unbalanced linguistic term sets 


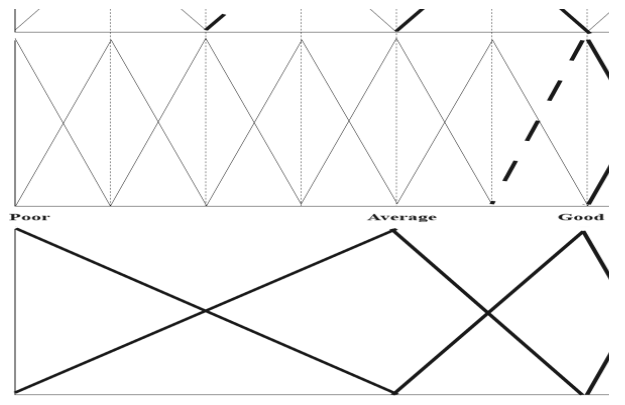

Fig. 3: Semantic representation of the sensory evaluation in $\mathrm{LH}$.

based on the 2-tuple computational model. To accomplish the processes of CW without loss of information dealing with $\mathrm{LH}$ and 2-tuples. The algorithm proposed in [10] builds a structure as the Table 3 with information that supports the computations with unbalanced labels (see Table 3). This table reports which label of the $L H$ represents a label $s_{i} \in S$ and additionally uses a boolean function noted as Brid to indicate when a label is represented by means of two different labels in the LH.

\begin{tabular}{|c||c|c|}
\hline$S$ & $L H(S)$ & $\operatorname{Brid}(S)$ \\
\hline$s_{0}=F$ & $s_{I(0)}^{G(0)}=s_{0}^{3}$ & False \\
\hline$s_{1}=D$ & $s_{I(1)}^{G(1)}=s_{1}^{3}$ & True \\
\hline$s_{2}=C$ & $s_{I(2)}^{G(2)}=s_{3}^{5}$ & True \\
\hline$s_{3}=B$ & $s_{I(3)}^{G(3)}=s_{7}^{9}$ & False \\
\hline$s_{4}=A$ & $s_{I(4)}^{G(4)}=s_{8}^{9}$ & False \\
\hline
\end{tabular}

Table 1: $L H(S)$ and $\operatorname{Brid}(S)$.

To accomplish the CW processes were introduced, two unbalanced linguistic transformation functions that converts a unbalanced linguistic term $s_{i} \in S$ into the linguistic term in the LH $s_{k}^{n(t)} \in L H=\bigcup_{t} l(t, n(t))$ and vice versa such a way the 2-tuple computational model can be used.

1. $L H$ : Transformation function that associates with each unbalanced linguistic 2-tuple $\left(s_{i}, \alpha\right)$, $s_{i} \in S$ its respective linguistic 2-tuple in $\mathrm{LH}$ $\left(s_{k}^{n(t)}, \alpha\right), s_{k}^{n(t)} \in L H$.

$L H:(S \times[0.5,-0.5)) \rightarrow(L H \times[0.5,-0.5))$ such that $\forall\left(s_{i}, \alpha_{i}\right) \in(S \times[0.5,-0.5)) \Longrightarrow$ $L H\left(s_{i}, \alpha_{i}\right)=\left(s_{I(i)}^{G(i)}, \alpha_{i}\right)$.

2. $L H^{-1}$ : Transformation function that associates with each linguistic 2-tuple expressed in LH its respective unbalanced linguistic 2-tuple in $S$.

$L H^{-1}:(L H \times[0.5,-0.5)) \rightarrow(S \times[0.5,-0.5))$, $\forall\left(s_{k}^{n(t)}, \alpha_{k}\right) \in(L H \times[0.5,-0.5)) \mid s_{k}^{n(t)} \in S^{n(t)}$, being $t$ a level of LH, then is defined by cases in $[10]$

\section{Unbalanced linguistic infor- mation sensory evaluation model}

The aim of this contribution is to propose a Sensory Evaluation model based on the linguistic decision analysis [9] to deal with unbalanced linguistic information whose mathematical formalism will be the linguistic 2-tuple model in order to obtain an evaluation framework where the experts can express their preferences in unbalanced linguistic term sets with different discrimination levels on both sides of the scale. The decision analysis scheme that will use our proposal for the sensory evaluation model consists of the following phases:

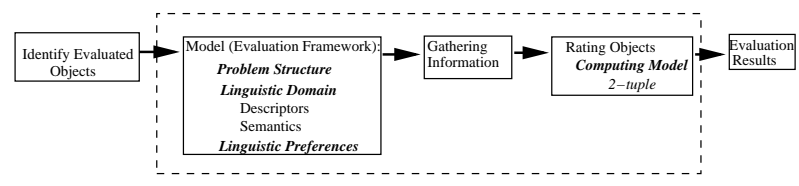

Fig. 4: Sensory Evaluation Scheme based on linguistic 2-tuple decision analysis.

- Identify Evaluated Objects

- Evaluation Framework: this phase defines the evaluation context in which the experts will express their preferences about the evaluated objects. Here it will be chosen the linguistic descriptors and their semantics.

- Gathering Information: the experts express their sensorial knowledge about the objects.

- Rating Objects: in this proposal the unbalanced linguistic computing model is used to rate the different objects. There must be chosen the aggregation operators that will be used 
in the rating process.

- Evaluation Results: The rates obtained in the before phase will serve to analyze the different evaluated objects.

The following subsections present in further detail the main phases of the linguistic sensory evaluation model proposed.

\subsection{Evaluation framework}

This phase defines the evaluation framework, such that, the problem structure is defined and the linguistic descriptors and semantics that will be used by the experts to provide their information about the sensory features of the evaluated objects are chosen. In some sensory evaluation problems could be that experts need a greater level of distinction in one side of the evaluation scale than in the other one, we propose a unbalanced linguistic evaluation framework where the experts can express their opinions in it. In this paper the linguistic decision analysis is based in a Multi-Experts Decision Making (MEDM) context [14]. Therefore the evaluation framework could be as:

- $E=\left\{e_{1}, \ldots, e_{n}\right\}$ panel of experts.

- $O=\left\{o_{1}, \ldots, o_{m}\right\}$ set of evaluated objects.

- $F=\left\{f_{1}, \ldots, f_{h}\right\}$, set of evaluated sensorial features for each object.

- $S=\left\{s_{0}, \ldots, s_{g}\right\}$, unbalanced linguistic term set

Applying the representation algorithm of unbalanced linguistic information to represent the unbalanced labels of $S=\left\{s_{0}\right.$ : $P($ Poor $), s_{1}: A($ Average $), s_{2}: G($ Good $), s_{3} \quad:$ $V G($ VeryGood $), s_{4}$ : E(Excellent $\left.)\right\}$. shown in Fig.1 whose semantics are obtained by the algorithm presented in [10] are those ones shown in Fig.3.

Furthermore, the algorithm provides information to control the representation of $S$ in the $\mathrm{CW}$ processes (see Table 3 ).

\subsection{Gathering information}

The experts provide their sensory subjective preferences using an unbalanced linguistic term set, $S$, fixed in the evaluation framework by means of utility vectors that contain a linguistic assessment for each evaluated feature.

- $e_{i}$ provides his/her preferences in $S$ by means of a utility vector:

$U_{i}=\left\{u_{11}^{i}, \ldots, u_{1 h}^{i}, u_{21}^{i}, \ldots, u_{2 h}^{i}, \ldots, u_{m 1}^{i}, \ldots, u_{m h}^{i}\right\}$ where $u_{j k}^{i} \in S$ is the assessment provided to the feature $f_{k}$ of the object $o_{j}$ by the expert $e_{i}$.

Due to the fact, that the evaluation model will use the unbalanced linguistic computing model the linguistic preferences provided by the experts will be transformed into linguistic 2-tuples according to the Remark 1.

\subsection{Rating objects}

Given that the semantics of the unbalanced term set belong to different levels of $L H$ we cannot operate directly with them. So we will unify the semantics of these labels in a level of the $L H$, called basic representation level $\left(t_{H G L S}\right)$ which will support the computation processes of unbalanced linguistic assessments [11]. We choose as $t_{H G L S}$ the level of $L H$ used in the representation algorithm which has associated the highest granularity label set (HGLS). We transform into $t_{H G L S}$ the preferences of the experts for every feature of the objects expressed in $S$ by means of the set of transformation functions $L H$ between levels of $L H$ presents in the subsection 2.2 .

1. Computing collective evaluations for each feature:

Once the unbalanced linguistic assessments are represented in $t_{H G L S}$, the rating process will compute a collective value for each feature, using an aggregation operator, $A G_{1}$, on the assessments provided by the experts represented in $t_{H G L S}$

$$
u_{j k}=A G_{1}\left(u_{j k}^{1}, \ldots, u_{j k}^{n}\right)
$$

2. Computing a collective evaluation for each object: the final aim of the rating process is to obtain a global evaluation, of each evaluated object according to all the experts and features that take part in the evaluation process. To do so, this process will aggregate the collective features values for each object using an aggregation operator, $A G_{2}$

$$
u_{j}=A G_{2}\left(u_{j 1}, \ldots, u_{j h}\right)
$$

The aggregation operators, $A G_{1}$ and $A G_{2}$, will depend on each evaluation problem taking into account if all experts or features are equally important or there are experts or features more important than the others.

The aggregation result is expressed in $S^{n\left(t_{H G L S}\right)}$. If we want to express the aggregation 
result expressed in $S$. This is achieved by applying the transformation function $L H^{-1}$ to the results obtained by $A G_{2}$.

\section{Conclusions}

The sensory evaluation is an evaluation process in which the information provided by the experts involves uncertainty because it is acquired via human senses. Therefore this information usually is vague and uncertain, could be that experts need a greater level of distinction in one side of the evaluation scale than in the other one.In this contribution we have presented a sensory evaluation model that offers an unbalanced linguistic evaluation framework to the experts in order to offer a greater flexibility to express their knowledge in the evaluation process.

\section{Acknowledgements}

This paper has been partially supported by the research project TIN2006-02121 and Feder Fonds.

\section{References}

[1] L.A. Zadeh. The concept of a linguistic variable and its applications to approximate reasoning. Information Sciences, Part I, II, III, 8,8,9:199-249,301-357,43-80, 1975.

[2] L. Martínez. Sensory evaluation based on linguistic decision analysis. International Journal of Aproximated Reasoning, 44 Num 2:148-164, 2007.

[3] G.B. Dijksterhuis. Multivariate Data Analysis in Sensory and Consumer Science, Food and Nutrition. Press Inc. Trumbull, Connecticut, USA, 1997.

[4] D. Ruan and X. Zeng (Eds.). Sensory Evaluation: Methodologies and Applications. Springer, 2004.

[5] H. Stone and J.L. Sidel. Sensory Evaluation Practice. Academic Press Inc., San Diego, CA, 1993.

[6] C.T. Chen. Applying linguistic decisionmaking method to deal with service quality evaluation problems. International Journal of Uncertainty, Fuzziness and Knowledge-Based Systems, 9(Suppl.):103-114, 2001.

[7] C.H. Cheng and Y. Lin. Evaluating the best main battle tank using fuzzy decision theory with linguistic criteria evaluation. European Journal of Operational Research, 142:174-186, 2002.
[8] L. Martínez, J. Liu, J.B. Yang, and F. Herrera. A multi-granular hierarchical linguistic model for design evaluation based on safety and cost analysis. International Journal of Intelligent Systems., 20(12):1161-1194, 2005.

[9] R.T. Clemen. Making Hard Decisions. An Introduction to Decision Analisys. Duxbury Press, 1995.

[10] F. Herrera, E. Herrera-Viedama, and L. Martínez. A fuzzy linguistic methodology to deal with unbalanced linguistic term sets. IEEE Transactions on Fuzzy Systems, page In press, 2007.

[11] F. Herrera and L. Martínez. A model based on linguistic 2-tuples for dealing with multigranularity hierarchical linguistic contexts in multiexpert decision-making. IEEE Transactions on Systems, Man and Cybernetics. Part B: Cybernetics, 31(2):227-234, 2001.

[12] F. Herrera and L. Martínez. A 2-tuple fuzzy linguistic representation model for computing with words. IEEE Transactions on Fuzzy Systems, 8(6):746-752, 2000.

[13] F. Herrera and L. Martínez. The 2-tuple linguistic computational model. Advantages of its linguistic description, accuracy and consistency. International Journal of Uncertainty, Fuzziness and Knowledge-Based Systems, 9(Suppl.):33-49, 2001.

[14] F. Herrera, E. Herrera-Viedma, and L. Martínez. A hierarchical ordinal model for managing unbalanced linguistic term sets based on the linguistic 2-tuple model. In Proceedings of the Eurofuse Workshop on Preference Modelling and Applications, pages 201-206, Granada (Spain), April 2001. 